\title{
Retrospective Audit of Compliance with Hospital Rib Fractures Pathway
}

\author{
K.S.Ang and F. Sage \\ Department of Anaesthesia, East Surrey Hospital, Redhill, United Kingdom.
}

\section{Background and Aims}

Rib fractures are associated with significant morbidity and mortality. Respiratory complications commonly occur in up to $31 \%$ of patients, and mortality rates as high as $33 \%$. The pathophysiology involves hypoventilation secondary to pain, damaged underlying lung and altered breathing mechanics. This further leads to retention of pulmonary secretions and pneumonia.

The mainstay of management is effective analgesia, early chest physiotherapy and ventilatory support where indicated. Amongst these strategies, pain management plays a key role. Effective analgesia enables deep breathing, adequate clearance of pulmonary secretions and compliance with chest physiotherapy.

In 2015, our hospital introduced a multidisciplinary rib fractures algorithm that helps identify patients at greatest risk of deterioration and provides a pathway that incorporates prompt analgesia, early referral to anaesthetics and critical care, chest physiotherapy and involvement of acute pain team to better manage these patients. All patients should be treated promptly according to the WHO analgesic ladder, with appropriate patients being considered for regional anaesthetic techniques.

This audit aims to investigate our compliance with hospital rib fractures pathway since its introduction.

\section{Methods}

Patients diagnosed with fractured ribs from January 2016 to April 2017 were identified using hospital-coding system. Data was collected retrospectively from case notes and compared with previous data collected before the algorithm's introduction. We recorded age, gender, number of broken ribs, analgesic regime, respiratory complications, critical care admission and hospital length of stay.

\section{Results}

Forty-six patients were identified (male: female ratio $=1: 0.84$ ). Median (range) age was $75.5(25-96)$ years, with 28 patients more than 65 years old. Median (range) number of fractured ribs was $3(1-9)$, with 16 patients suffered four or more fractured ribs. Analgesic regime prescribed in A\&E and in ward is depicted in Table I. Thirty patients (65\%) received regional anaesthesia for pain relief ( 29 serratus plane blocks, one intercostal block). Thirty-eight patients (83\%) received documented chest physiotherapy.

Nineteen patients suffered from chest infection. Thirteen patients were admitted to critical care, out of which seven required antibiotics for pneumonia. No patients required mechanical ventilation, however one patient was given supplementary nasal oxygen whilst two patients required high flow nasal oxygen therapy. Median (range) ICU/HDU length of stay was $3(2-16)$ days. Five patients died during their admission; all of them had concurring significant primary comorbidities. Median (range) hospital length of stay was 7 (I - 57) days.

Figure I demonstrates that since the pathway's introduction, there is significant increase in physiotherapy input, use of regional anaesthesia for opioid-sparing analgesia and acute pain team involvement.

Table 1 Analgesic regime prescribed in emergency department and in ward

\begin{tabular}{|c|c|c|c|c|}
\hline \multicolumn{6}{|c|}{ Analgesia in Emergency Department } \\
\hline Paracetamol & NSAIDs & Weak opioids & Strong opioids & None \\
\hline 33 & 8 & 18 & 23 & 2 \\
\hline \multicolumn{5}{|c|}{ Analgesia in Ward } \\
\hline Paracetamol & NSAIDs & Weak opioids & Strong opioids & None \\
\hline 43 & 12 & 21 & 39 & 1 \\
\hline
\end{tabular}

\section{Conclusion}

Our hospital pathway aims to improve patient outcomes and satisfaction following rib fractures. This audit demonstrates substantial improvements in our compliance since the pathway's introduction. Future direction includes evaluation of long-term outcomes such as chronic pain and disability.

Figure 1 Multidisciplinary approach to patient management pre and post hospital rib fracture pathway

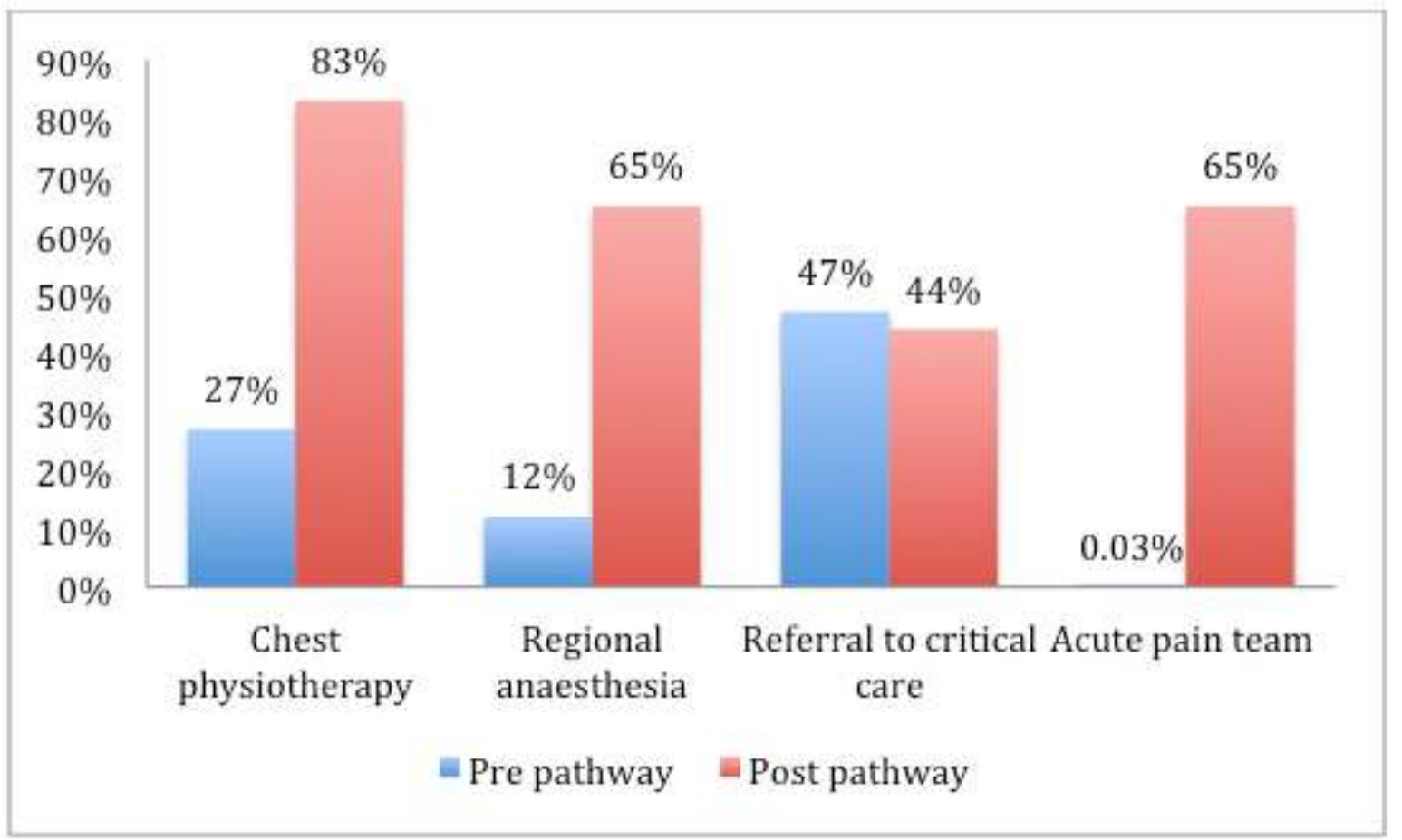

\section{References}

- Pressley $\mathbf{C}$ et al. Predicting outcome of patients with chest wall injury. Am J Surg 20 I2; 204: 910-4

- Easter A. Management of patients with multiple rib fractures. Am J Crit Care 200I; 10:320-9

- Flagel, BT et al. Half-a-dozen ribs: the breakpoint for mortality. Surgery 2005; 138: 7/ 7-723

- Kunhabdulla NP et al. Serratus anterior plane block for multiple rib fractures. Pain Physician 20 I 4; I 7: e65 I-e653 